MATHEMATICS OF COMPUTATION

Volume 68, Number 228, Pages 1749-1760

S 0025-5718(99)01126-6

Article electronically published on May 17, 1999

\title{
THE SECOND LARGEST PRIME DIVISOR OF AN ODD PERFECT NUMBER EXCEEDS TEN THOUSAND
}

\author{
DOUGLAS E. IANNUCCI
}

\begin{abstract}
Let $\sigma(n)$ denote the sum of positive divisors of the natural number $n$. Such a number is said to be perfect if $\sigma(n)=2 n$. It is well known that a number is even and perfect if and only if it has the form $2^{p-1}\left(2^{p}-1\right)$ where $2^{p}-1$ is prime.

No odd perfect numbers are known, nor has any proof of their nonexistence ever been given. In the meantime, much work has been done in establishing conditions necessary for their existence. One class of necessary conditions would be lower bounds for the distinct prime divisors of an odd perfect number.

For example, Cohen and Hagis have shown that the largest prime divisor of an odd perfect number must exceed $10^{6}$, and Hagis showed that the second largest must exceed $10^{3}$. In this paper, we improve the latter bound. In particular, we prove the statement in the title of this paper.
\end{abstract}

\section{INTRODUCTION}

For the natural number $n$, we denote the sum of its positive divisors by

$$
\sigma(n)=\sum_{d \mid n} d
$$

We define $n$ to be perfect if $\sigma(n)=2 n$. Euclid proved that if $2^{p}-1$ is prime then $2^{p-1}\left(2^{p}-1\right)$ is perfect; Euler showed that every even perfect number has this very same form. It is easily seen that primality for $p$ is necessary for the primality of $2^{p}-1$. Primes of the form $2^{p}-1$ are called Mersenne primes; hence there is a one-to-one correspondence between the even perfect numbers and the Mersenne primes. Exactly 37 Mersenne primes are known today. The question of whether they are infinite in number remains unanswered.

On the other hand, no odd perfect numbers have ever been discovered, nor has any proof of their nonexistence ever been given. Therefore, it is not known whether odd perfect numbers exist. In the meantime, many necessary conditions for their existence have been found. For example, the highest known lower bound for an odd perfect number, due to Brent, Cohen, and teRiele [4], is now $10^{300}$.

If a natural number $n$ has the unique prime factorization $\prod_{i=1}^{k} p_{i}^{a_{i}}$, we refer to $p_{1}^{a_{1}}, p_{2}^{a_{2}}, \ldots, p_{k}^{a_{k}}$, as the components of $n$. Cohen [6] showed that an odd perfect number must have a component exceeding $10^{20}$. One possible class of theorems giving necessary conditions for the existence of odd perfect numbers is

Received by the editor June 16, 1997 and, in revised form, August 25, 1997.

1991 Mathematics Subject Classification. Primary 11A25, 11Y70.

Key words and phrases. Perfect numbers, cyclotomic polynomials. 
$\mathcal{Q}(J)$. An odd perfect number has at least $J$ components.

Sylvester [21] gave the first theorem of this class in 1888 when he proved $\mathcal{Q}(5)$. Gradštein [9], Kühnel [16], and Webber [22] each independently proved $\mathcal{Q}(6)$. Likewise, Pomerance [18] and Robbins [20] independently proved $\mathcal{Q}(7)$. Hagis [10] subsequently proved $\mathcal{Q}(8)$, while Chein [5], in his doctoral dissertation, independently obtained the same result. It seems the amount of effort needed to prove $\mathcal{Q}(J)$ increases exponentially with $J$.

Pomerance [18] provides a lucid history of research on odd perfect numbers up to 1974. He speaks, in particular, of another possible class of theorems for odd perfect numbers, given by

$\mathcal{P}(K, M)$. An odd perfect number is divisible by $K$ distinct primes, each of which exceeds $M$.

In Section 2 of this paper, the arithmetic function $\sigma_{-1}$ is discussed. Upon considering the properties of $\sigma_{-1}$, one sees that proving $\mathcal{Q}(9)$ would entail far less work if one could first prove $\mathcal{P}(K, M)$ for as many $K$, and the largest $M$, as possible. The first known theorem of this class was given by Kanold [15] when he proved $\mathcal{P}(1,60)$ be elementary methods. With the aid of computation, Hagis and McDaniel [12] proved $\mathcal{P}(1,11200)$; the same authors [13] later proved $\mathcal{P}(1,100110)$. Condict [8] and Brandstein [3] proved $\mathcal{P}(1,300000)$ and $\mathcal{P}(1,500000)$, respectively. Finally, Cohen and Hagis [7] have proved $\mathcal{P}\left(1,10^{6}\right)$. We state this result as

Lemma 1. If $P$ is the largest prime divisor of an odd perfect number, then $P>$ $10^{6}$.

The first known theorem of the form $\mathcal{P}(2, M)$ was obtained by Pomerance [19] when he proved $\mathcal{P}(2,138)$. With the aid of computation, Hagis [11] later proved $\mathcal{P}\left(2,10^{3}\right)$. The purpose of this paper, then, is to prove $\mathcal{P}\left(2,10^{4}\right)$, which we state as

Theorem 1. If $S$ is the second largest prime divisor of an odd perfect number, then $S>10^{4}$.

In obtaining the main result of this paper, the method of proof used by Hagis [11] was modified and then applied. As with most recent results of this kind, computation plays a huge role in the proof of Theorem 1. All computations and computer searches for this paper were conducted on an IBM-486 personal computer using a UBASIC software package. Verification of all primes was carried out using the APR primality test, due to Adleman, Pomerance, and Rumely [1].

\section{SOME PRELIMINARIES}

Throughout this paper, positive (or nonnegative) integers are denoted by $a, b, c, \alpha$, $\beta, \gamma$, as well as by $h, i, j, k, l, m, n$, and by $H, I, J, K, L, M, N$. Primes, which are odd unless noted otherwise, are denoted by $\pi, p, q, r, s, t$, and by $P, Q, R, S, T$.

We say $p^{k} \| m$ if $p^{k} \mid m$ but ${ }^{k+1} \nmid m$. Next, $v_{p}(m)$ denotes the $p$-valuation of $m$; i.e., $v_{p}(m)=k$ if $p^{k} \| m$. If $p \nmid a$, we denote by $o_{p}(a)$ the exponent to which $a$ belongs, modulo $p$.

We denote by $\Phi_{m}(a)$ the cyclotomic polynomial, of order $m$, evaluated at $a$. Recalling the identity

$$
b^{n}-1=\prod_{d \mid n} \Phi_{d}(b),
$$


we see that

$$
\sigma\left(p^{a}\right)=\prod_{\substack{d \mid a+1 \\ d>1}} \Phi_{d}(p) .
$$

Hence, if $N$ is an odd perfect number with unique prime factorization given by $\prod_{i=1}^{k} p_{i}^{a_{i}}$, we have

$$
2 N=\prod_{i=1}^{k} \prod_{\substack{d \mid a_{i}+1 \\ d>1}} \Phi_{d}\left(p_{i}\right)
$$

From Theorems 94 and 95 in Nagell [17] we obtain

Lemma 2. For primes $p$ and $q, q \mid \Phi_{m}(p)$ if and only if $m=h q^{\gamma}$, where $h=o_{q}(p)$ and $\gamma \geq 0$. If $\gamma>0$, then $q \| \Phi_{m}(p)$.

An immediate consequence of (2) and Lemma 2 is

$$
v_{q}\left(\sigma\left(p^{a}\right)\right)= \begin{cases}v_{q}\left(\Phi_{h}(p)\right)+v_{q}(a+1), & \text { if } h \mid a+1, h>1, \\ v_{q}(a+1), & \text { if } h=1, \\ 0, & \text { otherwise. }\end{cases}
$$

Another immediate consequence of Lemma 2 is

Lemma 3. If $q \mid \Phi_{a}(p)$ and $r \mid \Phi_{b}(p)$, where $a \neq b, q \equiv 1(\bmod a)$, and $r \equiv 1(\bmod b)$, then $q \neq r$.

Bang [2] proved the following:

Lemma 4. If $m \geq 3$, then $\Phi_{m}(p)$ has a prime divisor $q$ with the property $q \equiv 1$ $(\bmod m)$.

Bang's actual result is stated in [18], and is stronger than Lemma 4.

The next result, originally due to Euler, gives the "shape" of an odd perfect number, by which is meant the nature of its unique prime factorization.

Lemma 5 (Euler's criterion for odd perfect numbers). An odd perfect number must have unique prime factorization given by

$$
\pi^{4 m+1} p_{1}^{2 a_{1}} p_{2}^{2 a_{2}} \cdots p_{k}^{2 a_{k}},
$$

where $\pi \equiv 1(\bmod 4)$.

In accordance with the terminology used in [18], we shall henceforth refer to $\pi$ as the special prime.

Let

$$
\sigma_{-1}(n)=\sum_{d \mid n} d^{-1}=\frac{\sigma(n)}{n} .
$$

Thus $n$ is perfect if and only if $\sigma_{-1}(n)=2$. Note that $\sigma_{-1}$ is multiplicative. Since $\sigma_{-1}\left(p^{a}\right)=1+1 / p+1 / p^{2}+\cdots+1 / p^{a}$, it is clear that

$$
\sigma_{-1}\left(p^{a}\right)<\sigma_{-1}\left(p^{b}\right)<\frac{p}{p-1} \text { if } a<b .
$$

If $p<q$ then $q /(q-1)<(p+1) / p$. Thus

$$
\sigma_{-1}\left(q^{b}\right)<\sigma_{-1}\left(p^{a}\right) \quad \text { for all } a \text { and } b, \text { if } p<q \text {. }
$$




\section{AcCeptable positive integers}

The proof of Theorem 1 will be given in Section 5, and will be by reductio ad absurdum. That is, we will assume the existence of an odd perfect number with exactly one prime divisor ${ }^{1} P>10^{4}$ and show that this assumption is untenable.

Now consider the 45-element ordered set of primes $X=\{1093,3851,3221,1423$, 4591, 9181, 6991, 1009, 5419, 127, 151, 19, 11, 3169, 4933, 467, 2801, 571, 4733, 7, $97,331,31,61,13,3,5,37,29,811,1621,307,17,23,1723,41,43,47,53,3541$, $59,67,5113,71,73\}$. We order $X$ from left to right so that 1093 precedes 3851 , 3851 precedes 3221,3221 precedes 1423 , and so forth.

Similarly, let

$$
\begin{array}{lll}
X_{1}=\{613,547,1093\}, & X_{2}=\{4603,179,3221\}, & X_{3}=\{1381,101,1009\}, \\
X_{4}=\{317,3169\}, & X_{5}=\{7489,2467,4933\}, & X_{6}=\{109,263,4733\}, \\
X_{7}=\{2557,5113\} . & &
\end{array}
$$

Again, we order $X_{i}$ from left to right for each $i, 1 \leq i \leq 7$. Finally, let

$$
Y=X \bigcup X_{1} \bigcup X_{2} \bigcup \cdots \bigcup X_{7},
$$

and denote, for $1 \leq i \leq 7$, the primes $p_{i}$ by

$$
\begin{aligned}
& p_{1}=1093, \quad p_{2}=3221, \quad p_{3}=1009, \quad p_{4}=3169, \\
& p_{5}=4933, \quad p_{6}=4733, \quad p_{7}=5113 .
\end{aligned}
$$

Note that $p_{i} \in X$, and $p \notin X$ if $p \in X_{i} \backslash\left\{p_{i}\right\}$, for $1 \leq i \leq 7$. We now give

Definition 1. For $p \in Y$ and $q<10^{4}$, let $h=o_{p}(q)$. We say the positive integer $k$ is $(p, q)$-acceptable if each of the following is true:

1. $k+1=h p^{\gamma}$, where $\gamma \geq 0,4 \nmid h$, and $2 \nmid h$ if $q \equiv 3(\bmod 4)$.

2. If $u$ precedes $p$ in $X$ (or $p_{i}$ in $X$ when $p \in X_{i}$ ), then $u \nmid \sigma\left(q^{k}\right)$.

3. $\sigma\left(q^{k}\right)$ has no prime factor between $10^{4}$ and $10^{6}$.

4. $\sigma\left(q^{k}\right)$ has at most one prime factor greater than $10^{6}$.

5. If $\sigma\left(q^{k}\right)$ has exactly one prime factor $R>10^{6}$, then there exists a prime $T$ (with $T=2$ considered only if $R \equiv 1(\bmod 4)$ ) such that all prime factors of $\Phi_{T}(R)$ are less than $10^{4}$ and none precedes $p$ in $X$ (or $p_{i}$ in $X$ when $p \in X_{i}$ ).

Note that $\Phi_{T}(R)$ has a prime factor greater than $10^{4}$ if $T>5000$, according to Lemma 4.

Let $\beta$ be the smallest positive integer such that $h p^{\beta} \geq 5000$. It follows by Lemmata 4 and 3 , by (2), and by conditions 3 or 4 of Definition 1 that $k$ is not $(p, q)$-acceptable if $k+1=h p^{\gamma}$ and $\gamma>\beta$. Thus the set of $(p, q)$-acceptable integers is finite.

A computer search was conducted and a list of all $(p, q)$-acceptable values was compiled. Exactly five were included which may or may not be $(p, q)$-acceptable; these correspond to the ordered triples $(k, p, q)$ given by $(1422,1423,5693),(1422$, $1423,8539),(4732,4733,9467),(810,811,8111)$, and $(810,811,9733)$. It was only determined in these cases that $\sigma\left(q^{k}\right)=p M$, where $M$ has no prime divisor less than $10^{9}$. As the magnitude of $M$ lay beyond the realm of capability of the UBASIC software package, these five values of $k$ where assumed $(p, q)$-acceptable for the indicated primes $p$ and $q$.

\footnotetext{
${ }^{1}$ Recall that $P>10^{6}$ by Lemma 1 .
} 
TABle 1 . The values $A_{p}, B_{p}$, and $C_{p}$, as defined in section 3 , for $p \in Y$.

\begin{tabular}{|llll|llll|llll|}
\hline \multicolumn{1}{|c}{$p$} & $A_{p}$ & $B_{p}$ & $C_{p}$ & $p$ & $A_{p}$ & $B_{p}$ & $C_{p}$ & $p$ & $A_{p}$ & $B_{p}$ & $C_{p}$ \\
\hline 613 & 5 & 1 & 0 & 317 & 0 & 1 & 0 & 37 & 6 & 1 & 1 \\
547 & 2 & 1 & 0 & 3169 & 1 & 1 & 0 & 29 & 0 & 1 & 2 \\
1093 & 5 & 0 & 0 & 7489 & 2 & 0 & 0 & 811 & 2 & 1 & 0 \\
3851 & 1 & 1 & 0 & 2467 & 1 & 1 & 0 & 1621 & 1 & 0 & 0 \\
4603 & 2 & 0 & 0 & 4933 & 2 & 0 & 0 & 307 & 2 & 1 & 0 \\
179 & 0 & 1 & 0 & 467 & 0 & 1 & 0 & 17 & 0 & 2 & 0 \\
3221 & 1 & 0 & 0 & 2801 & 1 & 0 & 0 & 23 & 0 & 1 & 0 \\
1423 & 4 & 1 & 0 & 571 & 2 & 1 & 1 & 1723 & 1 & 0 & 0 \\
4591 & 0 & 1 & 0 & 109 & 14 & 1 & 0 & 41 & 1 & 2 & 1 \\
9181 & 2 & 0 & 0 & 263 & 0 & 1 & 0 & 43 & 9 & 2 & 1 \\
6991 & 1 & 0 & 0 & 4733 & 2 & 0 & 0 & 47 & 0 & 1 & 0 \\
1381 & 1 & 0 & 0 & 7 & 142 & 4 & 2 & 53 & 0 & 1 & 0 \\
101 & 0 & 1 & 1 & 97 & 9 & 2 & 0 & 3541 & 1 & 0 & 0 \\
1009 & 1 & 1 & 0 & 331 & 3 & 1 & 1 & 59 & 0 & 2 & 0 \\
5419 & 1 & 0 & 0 & 31 & 31 & 1 & 2 & 67 & 1 & 1 & 0 \\
127 & 13 & 1 & 0 & 61 & 13 & 1 & 1 & 2557 & 0 & 1 & 0 \\
151 & 13 & 1 & 1 & 13 & 47 & 2 & 1 & 5113 & 1 & 0 & 0 \\
19 & 83 & 2 & 1 & 3 & 63 & 7 & 1 & 71 & 0 & 0 & 0 \\
11 & 13 & 2 & 2 & 5 & 0 & 4 & 1 & 73 & 2 & 0 & 0 \\
\hline
\end{tabular}

The complete list of all $(p, q)$-acceptable values, along with the corresponding factorizations of $\sigma\left(q^{k}\right)$, is too lengthy to be given here. Instead, a summary is presented in Table 1.

For each $p \in Y$, Table 1 gives three integers, each depending on $p$, which we call $A_{p}, B_{p}$, and $C_{p}$. They are defined as follows:

1. $A_{p}$ denotes the sum $\sum v_{p}\left(\sigma\left(q^{k}\right)\right)$, taken over all $k, q$, such that $k$ is even, $(p, q)$ acceptable, and $\sigma\left(q^{k}\right)$ contains no prime factors greater than $10^{4}$, or such that $(k, p, q)$ is one of the five ordered triples discussed above. For each $p \in Y$, and each $q<10^{4}$, it was found that there exists at most one $(p, q)$-acceptable value $k$.

2. $B_{p}$ denotes the maximum value of $v_{p}\left(\sigma\left(q^{k}\right)\right)$, taken over all $k, q$, such that $k$ is odd and $(p, q)$-acceptable.

3. $C_{p}$ denotes the maximum value of $v_{p}\left(\sigma\left(q^{k}\right)\right)$, taken over all $k, q$, such that $k$ is even, $(p, q)$-acceptable, and $Q \mid \sigma\left(q^{k}\right)$ where $Q>10^{6}$, but not if $(k, p, q)$ is one of the five ordered triples discussed above. For each $p \in Y$, it was found that, among all such factorizations of $\sigma\left(q^{k}\right)$, no prime $Q>10^{6}$ appeared more than once. That is to say, if $k_{1}$ is $\left(p, q_{1}\right)$-acceptable and $k_{2}$ is $\left(p, q_{2}\right)$-acceptable, $q_{1} \neq q_{2}$, and if $Q_{1}\left|\sigma\left(q_{1}^{k_{1}}\right), Q_{2}\right| \sigma\left(q_{2}^{k_{2}}\right)$, and $Q_{1}, Q_{2}>10^{6}$, then $Q_{1} \neq Q_{2}$.

\section{FeAsible PRIMES}

In the following definition, recall the set $Y, X, X_{1}, \ldots, X_{7}$, and the primes $p_{1}, \ldots, p_{7}$, as defined in Section 3. Also note that the prime $r$ in the following definition may assume the value 2 under certain circumstances. 
Definition 2. If $p \in Y$ we say the prime $r$ is $p$-feasible if each of the following is true:

1. $r \neq 2$ if $p \equiv 3(\bmod 4)$ or if $p=613,1381,101,317,7489,109$, or 2557 .

2. If $u$ precedes $p$ in $X$ (or, if $p \in X_{i}, u$ either precedes $p$ in $X_{i}$ or $p_{i}$ in $X$ ), then $u \nmid \Phi_{r}(p)$.

3. $\Phi_{r}(p)$ has no prime factor between $10^{4}$ and $10^{6}$.

4. $\Phi_{r}(p)$ has at most one prime factor greater than $10^{6}$.

5. If $\Phi_{r}(p)$ has exactly one prime factor $R>10^{6}$, then there exists a prime $T$ (with $T=2$ considered only if $R \equiv 1(\bmod 4)$ and $\left.p \notin X_{i} \backslash p_{i}\right)$ such that all prime factors of $\Phi_{T}(R)$ are less than $10^{4}$ and none precedes $p$ in $X$ (or, if $p \in X$, none precedes either $p$ in $X_{i}$ or $p_{i}$ in $X$ ).

6. $r \neq 3$ or $p \neq 3851$.

Now suppose $r$ is a prime such that condition 2 of Definition 2 is satisfied. Suppose further that $\Phi_{r}(p)=K M, q<10^{4}$ if $q \mid K, q>10^{4}$ if $q \mid M$, and $M>1$. Then either $r$ is not $p$-feasible, or $\Phi_{r}(p)=K Q^{m}, Q>10^{6}$. Let $h=o_{p}(Q)$. As seen in [19], if $K=1$, then $p \| \Phi_{h}(Q)$. For, in this case, we have $\Phi_{r}(p)=Q^{m}$, or

$$
1+p\left(1+p+p^{2}+\cdots+p^{r-2}\right)=Q^{m},
$$

and so $p \| Q^{m}-1$. This implies $h \mid m$, and thus $Q^{h}-1 \mid Q^{m}-1$. Hence $p \| \Phi_{h}(Q)$, according to (1) and Lemma 2 . We state this result as

Lemma 6. If $h=o_{p}(Q)$ and $\Phi_{r}(p)=Q^{m}$, then $p \| \Phi_{h}(Q)$.

We will then assume that $K>1$. Before proceeding, we state and prove an auxiliary result.

Lemma 7. For $m \geq 1, n \geq 1$, and $a \geq 1$, we have

$$
\left(1+a+a^{2}+\cdots+a^{n}\right)^{m} \equiv \sum_{k=0}^{n}\left(\begin{array}{c}
m+k-1 \\
k
\end{array}\right) a^{k}\left(\bmod a^{n+1}\right) .
$$

Proof. We use induction on $m$. The statement obviously holds when $m=1$. Assume it holds for some $m \geq 1$. Recall the identity

$$
\sum_{i=0}^{l}\left(\begin{array}{c}
m+i \\
i
\end{array}\right)=\left(\begin{array}{c}
m+l+1 \\
l
\end{array}\right)
$$

Thus the statement also holds for $m+1$, because

$$
\begin{aligned}
(1+a & \left.+a^{2}+\cdots+a^{n}\right)^{m+1} \\
& \equiv\left(\sum_{k=0}^{n} a^{k}\right)\left(\sum_{k=0}^{n}\left(\begin{array}{c}
m+k-1 \\
k
\end{array}\right) a^{k}\right)\left(\bmod a^{n+1}\right) \\
& \equiv \sum_{k=0}^{n} a^{k} \sum_{j=0}^{k}\left(\begin{array}{c}
m+j-1 \\
j
\end{array}\right)\left(\bmod a^{n+1}\right) \\
& \equiv \sum_{k=0}^{n}\left(\begin{array}{c}
m+k \\
k
\end{array}\right) a^{k}\left(\bmod a^{n+1}\right) .
\end{aligned}
$$

Let $H=o_{p}(K)$ and write

$$
K^{H} \equiv 1+c_{1} p+c_{2} p^{2}+\cdots+c_{r-1} p^{r-1}\left(\bmod p^{r}\right),
$$


where $^{2} 0 \leq c_{i}<p$ for $1 \leq i \leq r-1$. By Lemma 7 we have

$$
\begin{aligned}
\left(\Phi_{r}(p)\right)^{H} & =\left(1+p+p^{2}+\cdots+p^{r-1}\right)^{H} \\
& \equiv 1+\sum_{k=1}^{r-1}\left(\begin{array}{c}
H+k-1 \\
k
\end{array}\right) p^{k}\left(\bmod p^{r}\right) .
\end{aligned}
$$

Thus, raising both sides of the equation $\Phi_{r}(p)=K Q^{m}$ to the power $H$ yields

$$
Q^{m H}+Q^{m H} \sum_{k=1}^{r-1} c_{k} p^{k} \equiv 1+\sum_{k=1}^{r-1}\left(\begin{array}{c}
H+k-1 \\
k
\end{array}\right) p^{k}\left(\bmod p^{r}\right)
$$

so that for any $J, 1 \leq J \leq r-1$, we have

$$
Q^{m H}-1 \equiv \sum_{k=1}^{J}\left(\left(\begin{array}{c}
H+k-1 \\
k
\end{array}\right)-c_{k} Q^{m H}\right) p^{k}\left(\bmod p^{J+1}\right) .
$$

Hence $Q^{m H} \equiv 1(\bmod p)$, so that $h \mid m H$. Thus $Q^{h}-1 \mid Q^{m H}-1$, and it follows from (1) that $v_{p}\left(\Phi_{h}(Q)\right) \leq v_{p}\left(Q^{m H}-1\right)$.

Now suppose that $v_{p}\left(\Phi_{h}(Q)\right) \geq J+1$ for some $J, 1 \leq J \leq r-1$. Then $Q^{m H} \equiv 1$ $\left(\bmod p^{J+1}\right)$, so it follows from $(7)$ that

$$
p^{J+1} \mid \sum_{k=1}^{J}\left(\left(\begin{array}{c}
H+k-1 \\
k
\end{array}\right)-c_{k}\right) p^{k}
$$

Our arguments hold for any prime value of $Q$, even 2 , provided that $Q \nmid K$. Therefore we have proved

Lemma 8. Suppose $\Phi_{r}(p)=K M$, where $(K, M)=1$. Let $H=o_{p}(K)$ and $c_{k}=$ $\left[K^{H} / p^{k}\right]-p\left[K^{H} / p^{k+1}\right]$ for $1 \leq k \leq r-1$. Furthermore, suppose that

$$
p^{J+1} \nmid \sum_{k=1}^{J}\left(\left(\begin{array}{c}
H+k-1 \\
k
\end{array}\right)-c_{k}\right) p^{k} \text { for some } J, 1 \leq J \leq r-1 .
$$

Then the assumption that $M$ is a power of a prime (say, $M=Q^{m}$ ) leads to the conclusion that $v_{p}\left(\Phi_{h}(Q)\right) \leq J$ if $h=o_{p}(Q)$.

The reader may note that the case of Lemma 8 when $J=1$ appears in [11] as Lemma 2.

For each $p \in Y$, let us now define $\Psi(p)$ by the values given in Table 2 , which can be found in Section 5. The reader will note that $\Psi(p)$ is prime for all $p \in Y$. Upon consideration of the order in which the primes $p$ occur in $X$ and $X_{i}, 1 \leq i \leq 7$, it follows from Definition 2 and direct computation that if $r$ is $p$-feasible, then $r \geq \Psi(p)$.

If $r>5000$ and $p \in Y$, then it follows easily from Lemmata 2 and 4 that either condition 3 of Definition 2 is not satisfied, or $\Phi_{r}(p)=M$, where every prime factor of $M$ exceeds $10^{6}$. In the latter case, either $r$ is not $p$-feasible or $M=Q^{m}$, say, and $v_{p}\left(\Phi_{h}(Q)\right)=1\left(\right.$ where $\left.h=o_{p}(Q)\right)$ by Lemma 6 .

On the other hand, if $\Psi(p) \leq r<5000$, a computer search reveals that $\Phi_{r}(p)$ has a prime divisor exceeding $10^{4}$. Hence, in this case, we have $\Phi_{r}(p)=K M$, where $q<10^{4}$ if $q \mid K$ and $q>10^{4}$ if $q \mid M$, and where $M>1$. Again, either $r$

\footnotetext{
${ }^{2}$ Simply, for $1 \leq i \leq r-1$, put $c_{i}=\left[K^{H} / p^{i}\right]-p\left[K^{H} / p^{i+1}\right]$, where $[x]$ denotes the integer part of $x$. In other words, the $c_{i}$ are merely the digits obtained from the base- $p$ expansion of the integer $K^{H}$, and are uniquely determined.
} 
is not $p$-feasible, or $M=Q^{m}$ for some $Q>10^{6}$. If we assume the latter, then either $K=1$ or $K>1$. If $K=1$, then $v_{p}\left(\Phi_{h}(Q)\right)=1$ (where $h=o_{p}(Q)$ ) by Lemma 6. Otherwise, $K>1$. As in the statement of Lemma 8, let $H=o_{p}(K)$ and $c_{k}=\left[K^{H} / p^{k}\right]-p\left[K^{H} / p^{k+1}\right]$ for $1 \leq k \leq r-1$. If there exists $J$ such that $1 \leq J \leq r-1$ and $p^{J+1} \nmid \sum_{k=1}^{J}\left(\left(\begin{array}{c}H+k-1 \\ k\end{array}\right)-c_{k}\right) p^{k}$, then $v_{p}\left(\Phi_{h}(Q)\right) \leq J$ (where $\left.h=o_{p}(Q)\right)$ by Lemma 8. A computer search reveals that such a $J$ always exists.

We may now define an integer-valued function $f(p, r)$ over all $p \in Y$ and $r \geq$ $\Psi(p)$. If $r>5000$ we set $f(p, r)=1$. Otherwise $\Psi(p) \leq r<5000$, and by our preceding remarks we may write $\Phi_{r}(p)=K M$, where $q<10^{4}$ if $q \mid K$ and $q>10^{4}$ if $q \mid M$. In this case, set $f(p, r)=1$ if $K=1$. If $K>1$, then, letting $H=o_{p}(K)$ and $c_{k}=\left[K^{H} / p^{k}\right]-p\left[K^{H} / p^{k+1}\right]$ for $1 \leq k \leq r-1$, we find the minimum value $J$, $1 \leq J \leq r-1$, such that $p^{J+1} \nmid \sum_{k=1}^{J}\left(\left(\begin{array}{c}H+k-1 \\ k\end{array}\right)-c_{k}\right) p^{k}$; then we set $f(p, r)=J$. By our preceding remarks, the function $f(p, r)$ is well defined.

Finally, for all $p \in Y$, we let

$$
\omega(p)=\max _{r \geq \Psi(p)} f(p, r)
$$

The values for $\omega(p)$ may also be found in Table 2 in Section 5 .

\section{Proof of Theorem 1}

Without further explicit mention, we will let $N$ denote an odd perfect number, all of whose prime divisors, save one, are less than $10^{4}$. We let $P$ denote the largest prime divisor of $N$ and let $\alpha=v_{P}(N)$. We use the notation

$$
N=L P^{\alpha} \text {, }
$$

where $p<10^{4}$ if $p \mid L$. It follows from Lemma 1 that $P>10^{6}$. Thus

$$
2 N=\sigma(L) \sigma\left(P^{\alpha}\right) .
$$

Our first objective is to show that if $p \in X$, then $p \nmid N$. To do this, we must also show that if $p \in X_{i}$ and $p_{i}=\pi$ (recall that $\pi$ is the special prime), then $p \nmid N$.

We shall be dealing with the primes in $Y$ in the order in which they appear in Table 2. Suppose, then, that $p \mid N$, where either

1. $p \in X$ but $N$ is not divisible by $u$ if $u$ precedes $p$ in $X$, or

2. $p \in X_{i} \backslash\left\{p_{i}\right\}, p_{i}=\pi$, but $N$ is not divisible by $u$ if either $u$ precedes $p_{i}$ in $X$ or $u$ precedes $p$ in $X_{i}$.

We need some auxiliary results; for the first one given, recall the definitions of $A_{p}, B_{p}$, and $C_{p}$ given at the end of Section 3.

Lemma 9. Let $\lambda(p)=A_{p}+B_{p}+C_{p}$. Then $v_{p}(\sigma(L)) \leq \lambda(p)$.

Proof. Suppose $q^{a} \| L$ and $p \mid \sigma\left(q^{a}\right)$. Let $h=o_{p}(q)$. By (2), there exists $k$ such that $k+1 \mid a+1$ and $p \mid \Phi_{k+1}(q)$; hence $k+1=h p^{\gamma}, \gamma \geq 0$, by Lemma 5. Thus $\sigma\left(q^{k}\right) \mid \sigma\left(q^{a}\right)$ by (2). Note that conditions 2-5 of Definition 1 also hold for $k$; this follows from Lemma $5,(8),(9)$, and our assumption about $p$. Thus $k$ is $(p, q)$ acceptable. But, recalling our remarks concerning the description of $A_{p}$ (see the end of section 3), it follows that $k$ is uniquely determined by $p$ and $q$. Hence by (4), $v_{p}\left(\sigma\left(q^{a}\right)\right)=v_{p}\left(\sigma\left(q^{k}\right)\right)$. We may then write

$$
v_{p}(\sigma(L))=\sum_{i=1}^{c} v_{p}\left(\sigma\left(q_{i}^{a_{i}}\right)\right)=\sum_{i=1}^{c} v_{p}\left(\sigma\left(q_{i}^{k_{i}}\right)\right),
$$


TABle 2. $\lambda(p), \Psi(p)$, and $\omega(p)$ for $p \in Y$.

\begin{tabular}{|llll|llll|llll|}
\hline \multicolumn{1}{|c}{$p$} & $\lambda$ & $\Psi$ & $\omega$ & $p$ & $\lambda$ & $\Psi$ & $\omega$ & $p$ & $\lambda$ & $\Psi$ & $\omega$ \\
\hline 613 & 6 & 11 & 1 & 317 & 1 & 5 & 1 & 37 & 8 & 13 & 1 \\
547 & 3 & 7 & 1 & 3169 & 2 & 7 & 1 & 29 & 3 & 11 & 1 \\
1093 & 5 & 11 & 1 & 7489 & 2 & 7 & 1 & 811 & 3 & 11 & 2 \\
3851 & 2 & 7 & 1 & 2467 & 2 & 7 & 1 & 1621 & 1 & 5 & 1 \\
4603 & 2 & 5 & 1 & 4933 & 2 & 7 & 1 & 307 & 3 & 7 & 1 \\
179 & 1 & 5 & 1 & 467 & 1 & 5 & 1 & 17 & 2 & 11 & 2 \\
3221 & 1 & 5 & 1 & 2801 & 1 & 5 & 1 & 23 & 1 & 11 & 4 \\
1423 & 5 & 11 & 1 & 571 & 4 & 11 & 1 & 1723 & 1 & 5 & 1 \\
4591 & 1 & 5 & 1 & 109 & 15 & 19 & 1 & 41 & 4 & 11 & 3 \\
9181 & 2 & 5 & 1 & 263 & 1 & 5 & 1 & 43 & 12 & 17 & 1 \\
6991 & 1 & 5 & 1 & 4733 & 2 & 7 & 1 & 47 & 1 & 7 & 2 \\
1381 & 1 & 5 & 1 & 7 & 148 & 157 & 2 & 53 & 1 & 7 & 2 \\
101 & 2 & 7 & 2 & 97 & 11 & 17 & 1 & 3541 & 1 & 5 & 1 \\
1009 & 2 & 7 & 1 & 331 & 5 & 11 & 1 & 59 & 2 & 11 & 2 \\
5419 & 1 & 5 & 1 & 31 & 34 & 41 & 1 & 67 & 2 & 7 & 1 \\
127 & 14 & 19 & 2 & 61 & 15 & 23 & 2 & 2557 & 1 & 5 & 1 \\
151 & 15 & 19 & 1 & 13 & 50 & 59 & 2 & 5113 & 1 & 5 & 1 \\
19 & 86 & 97 & 2 & 3 & 71 & 89 & 6 & 71 & 0 & 5 & 2 \\
11 & 17 & 29 & 3 & 5 & 5 & 17 & 4 & 73 & 2 & 7 & 2 \\
\hline
\end{tabular}

where $k_{i}$ denotes that unique $\left(p, q_{i}\right)$-acceptable integer. Since $2 \mid \sigma\left(q^{k}\right)$ if $k$ is odd (Lemma 5), then at most one of the $k_{i}$ is odd. Also, by our remarks regarding the description of $C_{p}$ (see the end of Section 3), at most one of the $k_{i}$ can involve the divisibility of $\sigma\left(q_{i}^{k_{i}}\right)$ by a prime $Q>10^{6}$ (as $\sigma(L)$ itself is divisible by at most one such prime). The result follows directly from the definitions of $A_{p}, B_{p}$, and $C_{p}$.

Table 2 gives $\lambda(p)$ for all $p \in Y$ (recall the values $\Psi(p)$ and $\omega(p)$ from the previous section).

By (8), Lemma 5, and our assumption about $p$, it follows that $\Phi_{r}(p) \nmid 2 N$ if any of conditions $1-5$ of Definition 2 are not satisfied. Here, we show also that $\Phi_{3}(3851) \nmid N$ if $3851 \mid N$. For, suppose otherwise. Denoting by $Q$ the prime 1141081, we see that $\Phi_{3}(3851)=13 Q$ (thus $P=Q$ ). A finite computer search reveals that $\Phi_{T}(Q)$ is divisible by a prime exceeding $10^{4}$ for all odd $T$ (the search is finite because when $T>5000$, this follows from Lemma 4). Since this is not the case when $T=2$, we have, by $(3), \Phi_{2}(Q) \mid \sigma(N)$; hence $1693 \mid N$, as $1693 \mid \Phi_{2}(Q)$. Note that $Q$ would have to be the special prime. Thus, by (3), if $\Phi_{s}(1693) \mid 2 N$, then $s$ is odd. Another finite computer search reveals that $\Phi_{s}(1693)$ contains a prime factor, different from $Q$, which exceeds $10^{4}$ for all odd $s \geq 5$; the fact that $Q \nmid \Phi_{s}(1693)$ for odd $s$ follows from Lemma 2 since $o_{Q}(1693)=570540$. Thus, from $(3), 487 \mid N$ since $487 \mid \Phi_{3}(1693)$. Again, $487 \neq \pi$, and another finite computer search shows that $\Phi_{s}(487)$ contains a prime factor (different from $Q$ ) exceeding $10^{4}$ for all odd $s$ (note that $\left.o_{Q}(487)=380360\right)$. Hence $\Phi_{s}(487) \nmid N$ for all odd $s$, whereby (3) is contradicted.

We have shown that $\Phi_{r}(p) \nmid 2 N$ if $r$ is not $p$-feasible. Recalling Section 4 , we see from (2) that if $p^{a} \| N$ and $t$ is the smallest prime divisor of $a+1$, then $t \geq \Psi(p)$. We have thus proved 
Lemma 10. $v_{p}(N) \geq \Psi(p)-1$.

Recall that in Section 4 we remarked that $\Phi_{r}(p)$ has a prime divisor exceeding $10^{4}$ for all $r, \Psi(p) \leq r<5000$. By Lemma 4 , if $r>5000$, then $\Phi_{r}(p)$ has a prime divisor exceeding $10^{4}$. It follows from Lemma 10 that if $p^{a} \| N$, then $P \mid \sigma\left(p^{a}\right)$; therefore, $\sigma\left(p^{a}\right)=K P^{m}$, where $q<10^{4}$ if $q \mid K$. Let $h=o_{p}(P)$ and suppose $p^{b} \| \sigma\left(P^{\alpha}\right)$. Let $l=\left[\log _{p} 5000\right]$. It follows from Lemmata 3 and 4 and from (4) that $b \leq v_{p}\left(\Phi_{h}(P)\right)+l$. Since we assume $\sigma\left(p^{a}\right)=K P^{m}$, where $P \nmid K$, we have, by Lemma 8 and our definition of $\omega(p)$,

Lemma 11. $v_{p}\left(\sigma\left(P^{\alpha}\right)\right) \leq \omega(p)+\left[\log _{p} 5000\right]$.

We are finally able to proceed with the crux of the proof.

Now suppose $613^{a} \| N$ and $1093=\pi$. Recalling (8) and (9), by Lemmata 9 and 11 we have $a \leq \lambda(613)+\omega(613)+\left[\log _{613} 5000\right]=6+1+1=8$, but by Lemma 10 we have $a \geq \Psi(613)-1=11-1=10$. This contradiction proves that $613 \nmid N$ if $1093=\pi$.

Suppose $547^{a} \| N$ and $1093=\pi$. As in the preceding paragraph, and referring to Table 2 , we have $a \leq 3+1+1=5$, but also $a \geq 7-1=6$. Thus $547 \nmid N$ if $1093=\pi$.

Suppose $1093^{a} \| N$. Similarly, we have $a \leq 5+1+1=7$, but also $a \geq 11-1=10$. Thus $1093 \nmid N$.

In the same fashion, the reader may now prove the following facts, in succession, by referring to Table 2 and Lemmata 9, 11, and 10:

$3851 \nmid N, 4603 \nmid N$ if $3221=\pi, 179 \nmid N$ if $3221=\pi, 3221 \nmid N, 1423 \nmid N$, $4591 \nmid N, 9181 \nmid N, 6991 \nmid N, 1381 \nmid N$ if $1009=\pi, 101 \nmid N$ if $1009=\pi, 1009 \nmid N$, $5419 \nmid N, 127 \nmid N, 151 \nmid N, 19 \nmid N, 11 \nmid N, 317 \nmid N$ if $3169=\pi, 3169 \nmid N, 7489 \nmid N$ if $4933=\pi, 2467 \nmid N$ if $4933=\pi, 4933 \nmid N, 467 \nmid N, 2801 \nmid N, 571 \nmid N, 109 \nmid N$ if $4733=\pi, 263 \nmid N$ if $4733=\pi, 4733 \nmid N, 7 \nmid N, 97 \nmid N, 331 \nmid N, 31 \nmid N, 61 \nmid N$, $13 \nmid N, 3 \nmid N, 5 \nmid N, 37 \nmid N, 29 \nmid N, 811 \nmid N, 1621 \nmid N, 307 \nmid N, 17 \nmid N, 23 \nmid N$, $1723 \nmid N, 41 \nmid N, 43 \nmid N, 47 \nmid N, 53 \nmid N, 3541 \nmid N, 59 \nmid N, 67 \nmid N, 2557 \nmid N$ if $5113=\pi, 5113 \nmid N, 71 \nmid N, 73 \nmid N$.

Thus we have proved

Lemma 12. If $p \in X$, then $p \nmid N$.

It is now a simple matter to complete the proof of Theorem 1. From (8) and Lemma 12, it follows that $N$ has the form

$$
N=P^{\alpha} \prod_{i=1}^{k} q_{i}^{a_{i}}
$$

where $q_{i} \notin X, q_{i}<10^{4}$. Thus by (5) and (6)

$$
\sigma_{-1}(N)<\frac{10^{6}}{10^{6}-1} \prod_{\substack{q<10^{4} \\ q \notin X}} \frac{q}{q-1}<2 .
$$

This contradiction shows that $S>10^{4}$. Hence Theorem 1 is proved.

\section{Some CONCLUding REMARKS}

As mentioned in the Introduction, our bound on $S$, while perhaps in itself not of paramount interest, can nonetheless be quite useful for investigations concerning 
the number of components an odd perfect number may have, or its magnitude. This paper was motivated by the author's desire to publish his dissertation result [14], the proof of which is greatly shortened if the improved bound on $S$ is incorporated.

I had twice remarked, in Sections 4 and 5, that a computer search revealed that for $p \in Y, \Phi_{r}(p)$ contains a prime factor $Q>10^{4}$. Actually, this follows easily from Table 1 in [7] and a small number of calculations needed to cover the cases in Table 2 where $\Psi(p)=5$. The values for $\Psi(p)$ were chosen with this in mind, as well as Lemmata 10 and 11.

Finally, I would like to express my gratitude to my dissertation advisor, Peter Hagis, Jr., for taking the time to read an earlier draft of this paper. The astute comments and timely suggestions he gave me greatly improved its quality.

\section{REFERENCES}

1. L. Adleman, C. Pomerance, and R. Rumely, On distinguishing prime numbers from composite numbers, Ann. of Math. (2) 117 (1973), 173-206. MR 84e:10008

2. A. Bang, Taltheoretiske Undersøgelser, Tidsskrift Math. 5 IV (1886), 70-80, 130-137.

3. M. Brandstein, New lower bound for a factor of an odd perfect number, \#82T-10-240, Abstracts Amer. Math. Soc. 3 (1982), 257.

4. R. Brent, G. Cohen, and H. teRiele, Improved techniques for lower bounds for odd perfect numbers, Math. Comp. 57 (1991), 857-868. MR 92c:11004

5. E. Chein, An odd perfect number has at least 8 prime factors, Ph.D. Thesis, Pennsylvania State University (1979).

6. G. Cohen, On the largest component of an odd perfect number, J. Austral. Math. Soc., Ser. A 42 (1987), 280-286. MR 87m:11005

7. J. Condict, On an odd perfect number's largest prime divisor, Senior Thesis, Middlebury College (1978).

8. I. Gradštein, O. Nečetnych soveršennych čislah, Mat. Sb. 32 (1925), 476-510.

9. P. Hagis, Outline of a proof that every odd perfect number has at least eight prime factors, Math. Comp. 35 (1980), 1027-1032. MR 81k:10004

10. P. Hagis, On the second largest prime divisor of an odd perfect number, Analytic Number Theory, Lecture Notes in Mathematics, vol. 899, Springer-Verlag, Berlin and New York, 1981, pp. 254-263. MR 83i: 10004

11. P. Hagis and G. Cohen, Every odd perfect number has a prime factor which exceeds $10^{6}$, Math. Comp. 67 (1998), 1323-1330. MR 98k:11002

12. P. Hagis and W. McDaniel, On the largest prime divisor of an odd perfect number, Math. Comp. 27 (1973), 955-957. MR 48:3855

13. P. Hagis and W. McDaniel, On the largest prime divisor of an odd perfect number II., Math. Comp. 29 (1975), 922-924. MR 51:8021

14. D. Iannucci, On the third largest prime divisor of an odd perfect number, Doctoral Dissertation, Temple University (1995).

15. H.-J. Kanold, Folgerungen aus dem Vorkommen einer Gaußchen Primzahl in der Primfaktorenzerlegung einer ungeraden vollkommenen Zahl, J. Reine Angew. Math. 186 (1944), 25-29. MR 6:255c

16. U. Kühnel, Verscharfung der notwendigen Bedingungen für die Existenz von ungeraden vollkommenen Zahlen, Math. Z. 52 (1949), 202-211. MR 11:714b

17. T. Nagell, Introduction to number theory, Wiley, New York, 1951. MR 13:207b

18. C. Pomerance, Odd perfect numbers are divisible by at least seven distinct primes, Acta Arith. 25 (1974), 265-300. MR 49:4925

19. C. Pomerance, The second largest prime factor of an odd perfect number, Math. Comp. 29 (1975), 914-921. MR 51:8018

20. N. Robbins, The non-existence of odd perfect numbers with less than seven distinct prime factors, Ph.D. Thesis, Polytechnic Institute of Brooklyn (1972).

21. J. Sylvester, Sur l'impossibilité de l'existence d'un nombre parfait impair qui ne contient pas au moins 5 diviseurs premiers distincts, C. R. Acad. Sci. Paris 106 (1888), 522-526. 
22. G. Webber, Non-existence of odd perfect numbers of the form $3^{2 \beta} p^{\alpha} s_{1}^{2 \beta_{1}} s_{2}^{2 \beta_{2}} s_{3}^{2 \beta_{3}}$, Duke Math. J. 18 (1951), 741-749. MR 13:207e.

University of the Virgin Islands, 2 John Brewers Bay, St. Thomas, VI 00802

E-mail address: diannuc@uvi.edu 